\title{
Boundary tension: From wetting transition to prewetting critical point
}

Cite as: J. Chem. Phys. 102, 7584 (1995); https://doi.org/10.1063/1.469009

Submitted: 02 December 1994 . Accepted: 13 February 1995. Published Online: 31 August 1998

S. Perković, E. M. Blokhuis, E. Tessler, and B. Widom

\section{ARTICLES YOU MAY BE INTERESTED IN}

Critical point wetting

The Journal of Chemical Physics 66, 3667 (1977); https://doi.org/10.1063/1.434402

Mean-field density-functional model of a second-order wetting transition

The Journal of Chemical Physics 128, 114716 (2008); https://doi.org/10.1063/1.2895748

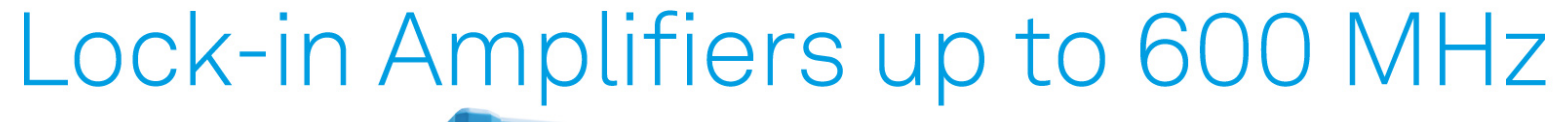

starting at

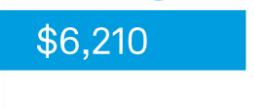

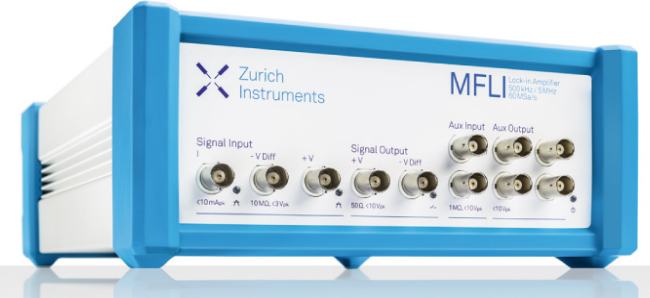

J. Chem. Phys. 102, 7584 (1995); https://doi.org/10.1063/1.469009

(c) 1995 American Institute of Physics.

102, 7584 


\title{
Boundary tension: From wetting transition to prewetting critical point
}

\author{
S. Perković, E. M. Blokhuis, E. Tessler, and B. Widom \\ Department of Chemistry, Baker Laboratory, Cornell University, Ithaca, New York 14853-1301
}

(Received 2 December 1994; accepted 13 February 1995)

\begin{abstract}
We develop a mean-field model free energy which we use in a van der Waals-like theory to study the prewetting transition in a system of two fluid phases when an incipient third phase may wet the interface between them. The line of prewetting transitions in the phase diagram is determined from the bulk wetting transition to the prewetting critical point. As the prewetting critical point is approached, the two coexisting surface phases become more and more alike, and they become identical at the prewetting critical point. The values of the boundary tension of the one-dimensional boundary formed by the edge-on meeting of two coexisting surface phases are calculated exactly (numerically) in a range between the wetting transition and the prewetting critical point. The data points obtained are extrapolated to a finite and positive boundary tension at the wetting transition and to a zero boundary tension at the prewetting critical point. These results are consistent with related earlier work. After scaling the dimensionless boundary tensions with appropriate force units, we determine that their values range from 0 at the prewetting critical point to $\mathscr{O}\left(10^{-12}\right) \mathrm{N}$ close to the wetting transition. These orders of magnitude compare well with recent experimental results. (C) 1995 American Institute of Physics.
\end{abstract}

\section{INTRODUCTION}

The prewetting transition is a coexistence of two surface phases of equal tensions but different structures. It is a $2 \mathrm{D}$ phenomenon analogous to a bulk phase equilibrium, where two bulk phases of different densities coexist. One of the surface phases consists of a microscopic layer of a third phase, which may become a bulk phase at three-phase coexistence, while the other surface phase has no such layer. In Fig. 1 we show a side view of the coexistence of two surface phases at the interface between two bulk fluid phases, $\alpha$ and $\gamma$. On the right side, the interface consists of a microscopic layer of a third $\beta$-like phase, while on the left side, the interface has no such layer. The two surface phases meet edge-on to create a one-dimensional boundary line.

The phenomenon of prewetting, along with its bulkphase counterpart, the wetting transition, are surface phase transitions, first predicted theoretically by $\mathrm{Cahn}^{1}$ and Ebner and Saam. ${ }^{2}$ The relation between these two phenomena is best described with the use of a generic phase diagram. According to the phase rule of Gibbs, for a three phase system, the wetting and prewetting transitions can only be described with a minimum of two components and two thermodynamic fields. In Fig. 2, the two thermodynamic fields, $\mu_{1}$ and $\mu_{2}$, may represent the temperature and the chemical potential difference between the system's two components. The solid curve represents thermodynamic states where the three bulk phases, $\alpha, \beta$ and $\gamma$, are at coexistence. Below the $W$ point on the coexistence curve, the system is partially wet: the $\beta$ phase forms a non-zero (and non- $180^{\circ}$ ) contact angle between the $\alpha$ and $\gamma$ phases. Above the $W$ point, the system is wet: the $\beta$ phase spreads at the $\alpha \gamma$ interface, so that there is no direct contact between the $\alpha$ and $\gamma$ phases. The $W$ point represents the wetting transition where the structure of the $\alpha \gamma$ interface changes from the partially wet to the wet state (or vice versa). If the wetting transition is a first-order surface phase transition, its first-order character manifests itself in the $\alpha \gamma$ two-phase region as well, on the left side of the coexistence curve. There, only the $\alpha$ and $\gamma$ phases coexist as bulks. Under certain conditions, the $\alpha \gamma$ interface consists of a microscopic layer of a $\beta$-like phase. That layer is not stable as a bulk, in coexistence with $\alpha$ and $\gamma$. The $\alpha \gamma$ interface is said to be prewet by $\beta$ (shaded region in Fig. 2). In other parts of the $\alpha \gamma$ two-phase region, such a structure is not present at the $\alpha \gamma$ interface. The coexistence of these two different structures of the $\alpha \gamma$ interface is a first-order surface phase transition: the prewetting transition. The loci of all the prewetting transitions create the prewetting line; the dashed curve in Fig. 2. That line is tangential to the three-phase coexistence line at the wetting transition, ${ }^{3}$ and ends at the prewetting critical point PCP, analogously to bulk phase criticality.

While theoretical calculations of the prewetting transition have been numerous since its prediction in 1977, its detection in simulations and experiments has been more recent. The first evidence for the existence of the prewetting line was obtained by Nicolaides and Evans ${ }^{4}$ in a Monte Carlo simulation of a confined lattice gas. In an extension of that work, they determined the 2D Ising-like character of the prewetting critical point. ${ }^{5}$ The first Monte Carlo simulation of an unconfined system was performed by Finn and Monson $^{6}$ who determined a prewetting transition in a model of fluid $\mathrm{Ar}$ and solid $\mathrm{CO}_{2}$. Velasco and Tarazona ${ }^{7}$ performed a density-functional calculation of the prewetting line for the solid-fluid model studied by Finn and Monson, and they found agreement for the surface critical point but not for the wetting temperature. Experimental evidence for the prewetting transition at a solid-fluid interface has been observed by Rutledge and Taborek, ${ }^{8}$ who have studied the prewetting phase transition of ${ }^{4} \mathrm{He}$ on Cs by using a quartz microbalance technique, and Ketola, Wang and Hallock, ${ }^{9}$ who used the technique of third sound to determine the prewetting transition in the same system. A prewetting transition has also been reported from adsorption and ellipticity measurements 


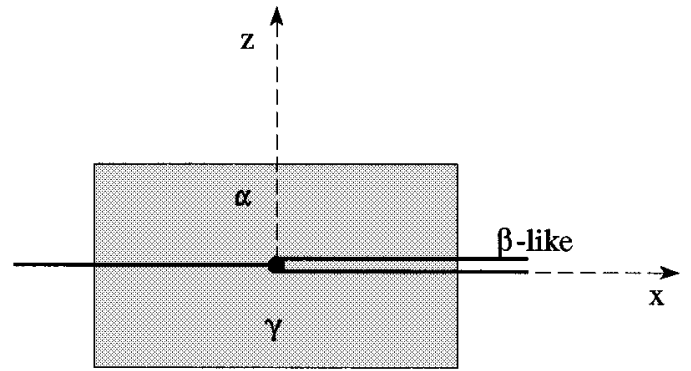

FIG. 1. A side view of two fluid surface phases coexisting at the prewetting transition. On the right, a microscopic layer of a $\beta$-like phase spreads at the $\alpha \gamma$ interface, while no such layer exists at the $\alpha \gamma$ interface on the left. The two interfaces meet edge-on to create the boundary line. The domain represents the area in which the system of Euler-Lagrange equations (4.2) and (4.3) are solved for the densities $\rho_{1}(x, z)$ and $\rho_{2}(x, z)$.

at the interface of the silica/water-2,6-lutidine mixture, ${ }^{10}$ and from adsorption measurements, in the silica-water2,5-lutidine system. ${ }^{11}$ Only recently has there been unambiguous evidence for the prewetting transition in fluid-fluid interfaces. Kellay, Bonn and Meunier ${ }^{12}$ presented evidence for the existence of a prewetting transition in a binary liquid mixture of methanol and cyclohexane. They have confirmed the tangential approach of the prewetting line to the threephase coexistence line at the wetting transition, as well as the 2D Ising-like character of the prewetting critical point.

In this paper, we develop a model mean-field excess free-energy density, that is a functional of the system's densities, in order to study the prewetting transition and calculate the boundary tension of the one-dimensional boundary line that is created by the edge-on meeting of the two coex-

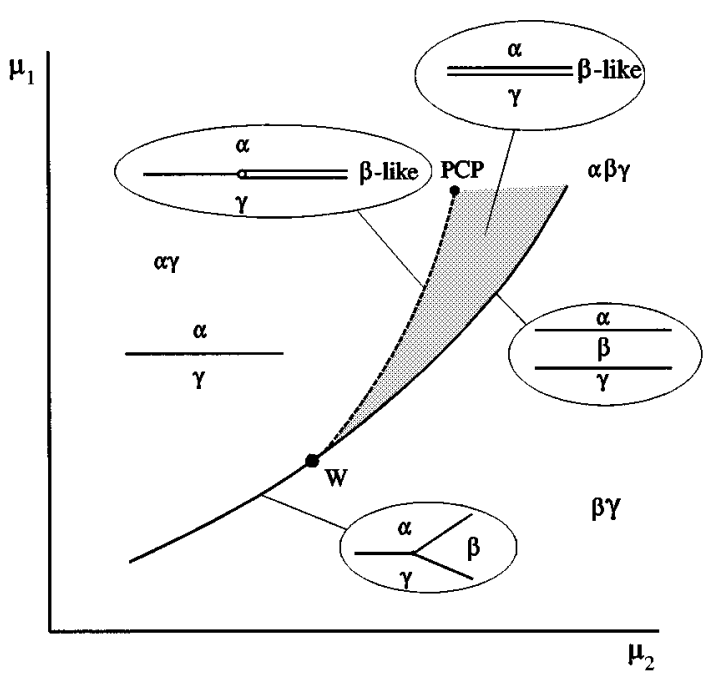

FIG. 2. Generic phase diagram of a system of three phases. $\mu_{1}$ and $\mu_{2}$ are any two thermodynamic fields such as the temperature and chemical potential difference of the two components. The solid curve is the three-phase coexistence curve. The point $W$ represents the wetting transition. Below the point $W$, on the solid curve, in the stable states, the $\alpha \gamma$ interface is partially wet, and above it, it is wet. The dashed curve, called the prewetting line, represents the loci of prewetting transitions, where two different structures of the $\alpha \gamma$ interface coexist. The prewetting line meets the three-phase coexistence line tangentially at the wetting transition $W$, and terminates at the other end in a prewetting critical point, PCP. isting surface phases (fig. 1). The boundary tension $\tau_{b}$ is the excess free energy associated with the density inhomogeneity in the boundary line, per unit length of that line. In the van der Waals-like theory that we employ, ${ }^{13}$ the boundary tension $\tau_{b}$ is defined as:

$$
\tau_{b}=\underset{\rho_{1}, \rho_{2}}{\min }\left[\int_{-\infty}^{\infty} d x\left[\int_{-\infty}^{\infty} d z \quad \Psi\right]-\sigma\right],
$$

where $\Psi$ is the model excess free-energy density that will be defined in Sec. II and $\sigma$ is the surface tension of the twodimensional interface and is given by:

$$
\sigma=\min _{\rho_{1}, \rho_{2}} \int_{-\infty}^{\infty} d z \Psi, \quad(x \rightarrow \pm \infty) .
$$

In Eq. (1.1), $\Psi$ is a function of $x$ and $z$, the directions parallel and perpendicular to the interface, respectively (fig. 1). In the limits of $x \rightarrow \pm \infty$, i.e. very far from the boundary line region, the functional $\Psi$ becomes independent of $x$. It is that functional that is used in Eq. (1.2). The functions $\rho_{1}$ and $\rho_{2}$ are the density profiles of the system's two components. In Eq. (1.1), they are two-dimensional, while in Eq. (1.2), they depend only on the $z$ coordinate.

Related to the boundary tension $\tau_{b}$ is the line tension $\tau$ which is defined analogously to $\tau_{b}$ in Eq. (1.1). The line tension $\tau$ is the excess free energy due to the inhomogeneities in the three-phase contact line in the partially wet state (states below the $W$ point on the three-phase coexistence curve in fig. 2), per unit length of that line. The line and boundary tensions become equal at the wetting transition. ${ }^{14,15}$

Here we present numerical calculations (that are exact for the model studied in the mean-field approximation) of the boundary tension for a system of two fluid phases. The case of a fluid on a solid substrate has been investigated recently. ${ }^{16}$ We determine $\tau_{b}$ over the whole length of the prewetting line, from the wetting transition to the prewetting critical point. The boundary tension data points are extrapolated with a form that is analogous to the one obtained by Indekeu $^{17}$ (see also Ref. 16) and by Varea and Robledo ${ }^{18}$ to give a finite and positive $\tau_{b}$ at the wetting transition. The vanishing of the boundary tension at the prewetting critical point is described with a mean-field exponent. Furthermore, the surface phases are studied as the prewetting critical point is approached. For completeness, we perform this analysis on a system of a fluid phase on a solid substrate as well, studied in detail by Blokhuis ${ }^{19}$ and Perković, Blokhuis and Han, ${ }^{16}$ within the van der Waals theory. Finally, we determine orders of magnitude for the boundary tensions, for the system of two fluid phases and the system of a fluid phase on a substrate, and compare them to experimental results for the boundary tension of the boundary between two lipid monolayer domains at the air-water interface. ${ }^{20}$

\section{MODEL FREE ENERGY}

In this section, we define the model excess free-energy density used to study the prewetting transition in a system of two fluid phases, with a van der Waals-like theory. ${ }^{13}$ That 
theory postulates the existence of a free-energy density $\Psi$ which depends on the local densities of the system's components and on the spatial derivatives of these densities at the same point.

Consider a system consisting of three fluid phases, $\alpha$, $\beta$ and $\gamma$, where $\beta$ is either a bulk phase at coexistence with $\alpha$ and $\gamma$, or an incipient layer at the $\alpha \gamma$ interface. For such a system, $\Psi$ is a functional of the two densities $\rho_{1}$ and $\rho_{2}$ of the system's two components:

$$
\Psi\left(\rho_{1}, \rho_{2}\right)=F\left(\rho_{1}, \rho_{2}\right)+\frac{1}{2}\left(\left(\nabla \rho_{1}\right)^{2}+\left(\nabla \rho_{2}\right)^{2}\right),
$$

with

$$
\begin{aligned}
F= & 16 \rho_{2}^{2}\left(\rho_{2}-b\right)^{2}+\epsilon\left(\rho_{1}^{2}-1\right)^{2}+\left[\rho_{2}-c\left(\rho_{1}+1\right)\right]^{2}\left[\rho_{2}\right. \\
& \left.+b\left(\rho_{1}-1\right)\right]^{2}+\left[\rho_{2}+c\left(\rho_{1}-1\right)\right]^{2}\left[\rho_{2}-b\left(\rho_{1}+1\right)\right]^{2} .
\end{aligned}
$$

$\Psi$ is the free-energy density associated with the presence of inhomogeneous regions, in excess of the free-energy density in the bulk phases. It vanishes in the bulk phases, and has a positive value within inhomogeneous regions, such as the two-dimensional interface and the boundary line. The freeenergy density $F$ is the local free-energy that is in excess from the free-energy in the bulk phases when the density is constant in the neighbourhood of the local point. The gradient terms account for the free-energy excess associated with the variation in density in the inhomogeneous regions. The $\nabla$ gradient operator in Eq. (2.1) is one-dimensional in the $z$-direction (fig. 1), if one is far from the boundary line region $(x \rightarrow \pm \infty)$ so that $\rho_{1}$ and $\rho_{2}$ are functions of $z$ only. It is two-dimensional in the $x$ and the $z$ directions (fig. 1), when one is close to the boundary line region.

The contour lines of the excess free-energy density $F$ in Eq. (2.2), i.e. lines of constant free-energy density, are plotted in Fig. 3a as a function of the densities $\rho_{1}$ and $\rho_{2}$ (solid curves). The values of the three phenomenological parameters $b, \epsilon$ and $c$ are fixed at $b=0.50, \epsilon=0.1553$ and $c=-0.7$. These parameters represent three thermodynamic fields, such as the chemical potentials of the system's two components and the temperature. The two trajectories in fig. $3 \mathrm{a}$ (dashed curves) represent the variation of $\rho_{1}$ with $\rho_{2}$, along the $z$ coordinate (fig. 1), for the two different structures of the $\alpha \gamma$ interface, coexisting at the prewetting transition. This will be discussed further in Sec. III. In Fig. 3b, we plot the same figure as in Fig. 3a, but with equal $\rho_{1}$ and $\rho_{2}$ scales, and showing only $\rho_{1}>0$. The model is symmetric, so for $\rho_{1}<0$, one has the mirror image of fig. $3 \mathrm{~b}$. The trajectories are tangent to the long axes of the elliptical contours around $\left(\rho_{1}, \rho_{2}\right)=(1,0)$ (shown in fig. 3b) and $\left(\rho_{1}, \rho_{2}\right)=(-1,0)$ (by symmetry). The slight tilt from horizontal of the long axes of these ellipses (although hardly visible on the scale of this plot) is due to the parameter $c=-0.7$. For larger absolute values of $c$ the tilt is greater. The field $c$ has to be different from 0 , since only then are the elliptical contours around $\left(\rho_{1}, \rho_{2}\right)=(1,0)$ and $\left(\rho_{1}, \rho_{2}\right)=(-1,0)$ tilted. This tilt in the contour lines enables the structures of the two $\alpha \gamma$ surface phases to become identical, thus producing a prewetting critical point. If $c=0$, the tilt in the elliptical contours disappears, and there is no prewetting critical point in the model $F$

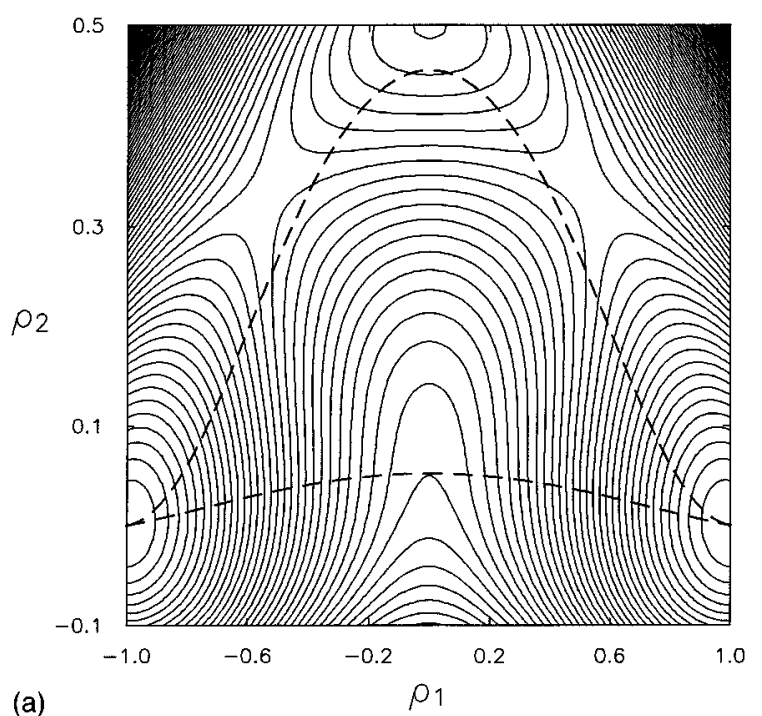

(a)

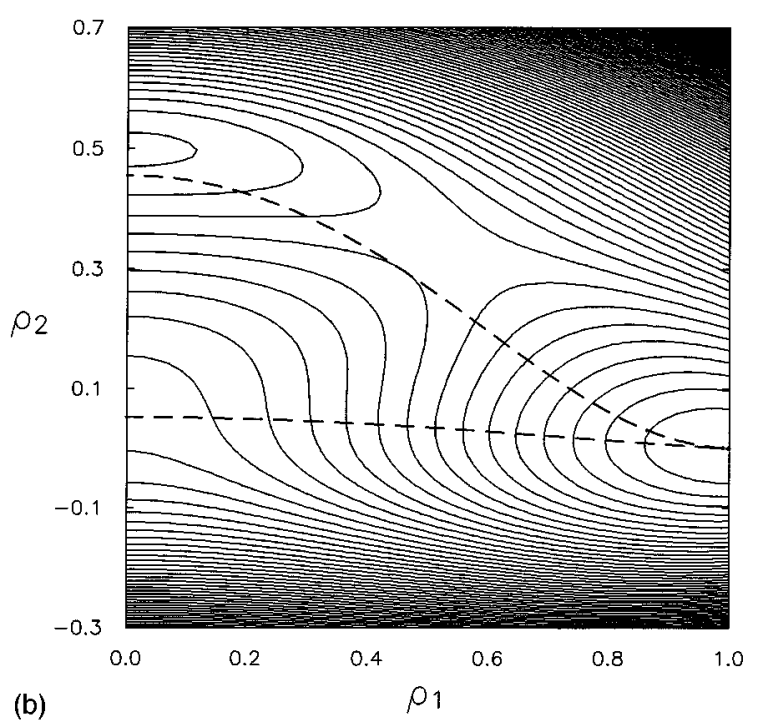

FIG. 3. (a) Plot of the contours of $F$ from Eq. (2.2) (solid curves) with two trajectories (dashed curves) representing coexistence of the two fluid surface phases at the prewetting transition for $b=0.50, \epsilon=0.1553$ and $c=-0.7$ (b) Same as in (a), but with equal $\rho_{1}$ and $\rho_{2}$ scales and for $\rho_{1}>0$ only ( $\rho_{1}<0$ is its mirror image). The trajectories are tangential to the long axes of the elliptical contours centered at the values of $\rho_{1}$ and $\rho_{2}$ for the $\alpha$ and $\gamma$ phases. Note that the contour interval has been changed between Figs. 3a and $3 b$.

in Eq. (2.2). Here, we only consider the case where $b>0$ and $c<0$ (analogous results would be obtained for $b<0$ and $c>0$ ). The field $\epsilon$ in Eq. (2.2) is a measure of the distance from the three-phase coexistence curve. For $\epsilon=0$, the system consists of three bulk phases at coexistence, while for $\epsilon>0$, only the $\alpha$ and $\gamma$ phases are at bulk equilibrium. This point is discussed in more detail below. The densities, distances and free energies in Eqs. (2.1) and (2.2) are scaled so that they are all dimensionless. Furthermore, the densities $\rho_{1}$ and $\rho_{2}$ are relative densities, and therefore can be negative.

When $\epsilon=0$, the excess free-energy density $F$ is positive for all values of the densities $\rho_{1}$ and $\rho_{2}$ except for those 
values that describe the bulk $\alpha, \beta$ and $\gamma$ phases. These densities are given by:

$$
\begin{aligned}
& \left(\rho_{1}^{\alpha}, \rho_{2}^{\alpha}\right)=(1,0), \\
& \left(\rho_{1}^{\beta}, \rho_{2}^{\beta}\right)=(0, b), \\
& \left(\rho_{1}^{\gamma}, \rho_{2}^{\gamma}\right)=(-1,0),
\end{aligned}
$$

where $\left(\rho_{1}^{\alpha}, \rho_{2}^{\alpha}\right)$ are the densities of components 1 and 2 in the bulk $\alpha$ phase, $\left(\rho_{1}^{\beta}, \rho_{2}^{\beta}\right)$ are the densities of components 1 and 2 in the bulk $\beta$ phase and $\left(\rho_{1}^{\gamma}, \rho_{2}^{\gamma}\right)$ are the densities of components 1 and 2 in the bulk $\gamma$ phase. At these values of $\rho_{1}$ and $\rho_{2}, F=0$ and its partial derivatives with respect to $\rho_{1}$ and $\rho_{2}$ vanish as well.

The form of the free energy in Eq. (2.2) is chosen for reasons of mathematical simplicity and convenience. It is not necessary to have a model of the free energy in which the density of one component is equal in both phases while the density of the other component is different in both phases. The same results would be produced with different definitions of the densities in the bulk phases.

When $\epsilon>0$, the excess free-energy density $F$ is still 0 and has vanishing partial derivatives with respect to $\rho_{1}$ and $\rho_{2}$ in the $\alpha$ and $\gamma$ phases, but now $F$ has a local minimum that is positive $(F=\epsilon)$ for the values of $\left(\rho_{1}^{\beta}, \rho_{2}^{\beta}\right)=(0, b)$. These values of $\rho_{1}$ and $\rho_{2}$ describe the bulk $\beta$ phase when $\epsilon=0$. When $\epsilon>0$, the $\beta$ phase is unstable as a bulk and it does not coexist with the $\alpha$ and $\gamma$ phases, since its free energy is slightly higher than the free energies of the $\alpha$ and $\gamma$ phases. The system is off the three-phase coexistence line, and is in the two-phase $\alpha \gamma$ region in fig. 2 .

As was mentioned in Sec. I, two thermodynamic fields are sufficient to describe a system of three bulk phases undergoing wetting and prewetting transitions. For that reason, we keep one of the thermodynamic fields in our model free energy Eq. (2.2) constant: $c=-0.7$, and we only vary the values of $b$ and $\epsilon$ in order to determine the prewetting line in the phase diagram. The value of $c$ has to be different from 0 , for the reason discussed above.

\section{PREWETTING TRANSITION AND SURFACE PHASES}

The prewetting transition in a system of fluid phases is the coexistence of two fluid surface phases of equal tension but of different density profiles. One of the surface phases (the $\alpha \gamma^{*}$ surface phase) consists of a microscopically thick layer of an incipient phase, the $\beta$-like layer in fig. 1 , at the interface between the bulk $\alpha$ and $\gamma$ phases, while the other surface phase (the $\alpha \gamma$ surface phase) has no such layer. If $\sigma^{\alpha \gamma}$ is the surface tension of the $\alpha \gamma$ surface phase, while $\sigma^{\alpha \gamma *}$ is the surface tension of the $\alpha \gamma^{*}$ surface phase, the condition for the prewetting transition is given by

$$
\sigma^{\alpha \gamma}=\sigma^{\alpha \gamma *} .
$$

The equilibrium surface tension and equilibrium density profiles of the $\alpha \gamma$ interface are obtained by minimizing the integral of the excess free-energy density $\Psi\left(\rho_{1}, \rho_{2}\right)$ given in Eq. (2.1), with respect to the densities $\rho_{1}$ and $\rho_{2}$, far away from the boundary line region $(x \rightarrow \pm \infty$ in fig. 1$)$,

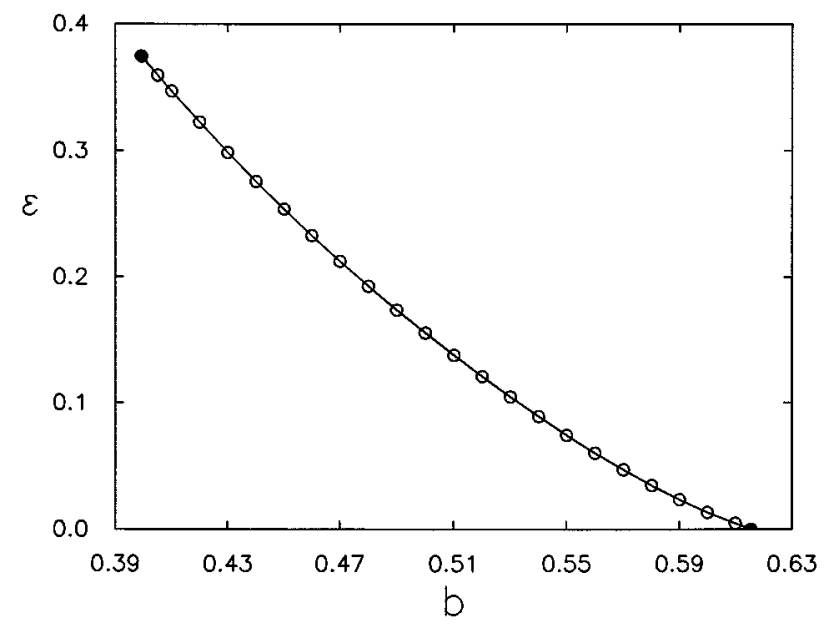

FIG. 4. Plot of the prewetting line in the $\epsilon, b$ thermodynamic space, for $c=-0.7$. The wetting transition is at $b=b_{w}=0.6175$ and $\epsilon=0$ (filled circle). The prewetting line terminates at the prewetting critical point $b=b_{\text {crit }}=0.3993$ and $\epsilon=\epsilon_{\text {crit }}=0.3745$ (filled circle).

$$
\sigma=\min _{\rho_{1}, \rho_{2}} \int_{-\infty}^{\infty} d z \Psi\left(\rho_{1}, \rho_{2}\right), \quad(x \rightarrow \pm \infty),
$$

where $z$ is the direction perpendicular to the interface. The obtained $\sigma$ is a function of the three thermodynamic fields, $b, c$ and $\epsilon$. At the prewetting transition, the surface tension of the $\alpha \gamma$ and $\alpha \gamma *$ surface phases are equal for the same set of thermodynamic fields $b, c$ and $\epsilon$,

$$
\sigma^{\alpha \gamma}(b, c, \epsilon)=\sigma^{\alpha \gamma *}(b, c, \epsilon) .
$$

Both $\sigma^{\alpha \gamma}(b, c, \epsilon)$ and $\sigma^{\alpha \gamma *}(b, c, \epsilon)$ are obtained numerically, using a conjugate gradient optimization algorithm. ${ }^{21}$ The conjugate gradient method is an iterative method that minimizes a function $p$ of $N$ variables by starting at a point $p(N)$ in the function's space and performing $N$ line minimizations along the directions of mutually conjugate vectors. These directions are "non-interfering" directions, i.e minimization along one of the directions is not invalidated by subsequent minimization along another direction. Furthermore, a separate evaluation of the gradients allows for a decrease in the number of minimizations required, from $N^{2}$ to $N .^{21}$ In order to use the conjugate gradient method, the integral in Eq. (3.2) is approximated by a sum over 257 (this number is important for the calculation of the boundary tension in Sec. IV) evenly spaced points in the interval $-10 \leqslant z \leqslant 10$, where $z$ is scaled so that it is dimensionless. We have used other intervals as well: $-5 \leqslant z \leqslant 5$ with 129 points so that the spacing is the same as in the above case, and $-6 \leqslant z \leqslant 6$ with 257 points, so that the spacing is smaller, with no significant differences in the results. The values of the densities $\rho_{1}$ and $\rho_{2}$ at the end points of the interval are chosen to be those of the bulk $\alpha$ and $\gamma$ phases: $\left(\rho_{1}^{\alpha}, \rho_{2}^{\alpha}\right)=(1,0)$ and $\left(\rho_{1}^{\gamma}, \rho_{2}^{\gamma}\right)=(-1,0)$. The sum is then minimized with respect to 255 values of $\rho_{1}$ and 255 values of $\rho_{2}$. During the minimization process, we have kept $c$ constant at $c=-0.7$ and we have fixed the value of $b$. Then, we determined $\sigma^{\alpha \gamma}$ and $\sigma^{\alpha \gamma *}$ for a set of $\epsilon$ values. The prewetting transition is then determined graphically by locating the value of $\epsilon$ for which Eq. (3.3) is valid. In fig. 4, 
we show a plot of the prewetting line determined by the above method. The axes are the thermodynamic fields $\epsilon$ and $b$, while $c=-0.7$. The data points, connected by a smooth curve, represent states where two different structures of the $\alpha \gamma$ interface coexist. Below the prewetting line, and for $\epsilon>0$, the thermodynamically stable states are the ones where the $\alpha \gamma$ interface is prewet by the $\beta$-like layer. Above the prewetting line, the non-prewet state is thermodynamically stable. The three bulk phases $\alpha, \beta$ and $\gamma$ coexist at $\epsilon=0$. For $b<b_{w}$, the $\beta$ phase wets the $\alpha \gamma$ interface, and it does not wet it for $b>b_{w}$, where $b_{w}=0.6175$ is the value of $b$ at which the first-order wetting transition occurs.

The value of $b$ at the wetting transition, $b_{w}$, is determined with the same conjugate gradient algorithm that was used for the determination of the prewetting line. Going back to the phase diagram in fig. 2 , the partially wet state (below the $W$ point on the three-phase coexistence curve) is mechanically stable when ${ }^{22}$

$$
\sigma^{\alpha \gamma}<\sigma^{\alpha \beta}+\sigma^{\beta \gamma},
$$

where $\sigma^{\alpha \gamma}$ is the surface tension of the $\alpha \gamma$ interface, $\sigma^{\alpha \beta}$ is the surface tension of the $\alpha \beta$ interface and $\sigma^{\beta \gamma}$ is the surface tension of the $\beta \gamma$ interface. The wet state (above the $W$ point on the three-phase coexistence curve in fig. 2) is stable when ${ }^{22}$

$$
\sigma^{\alpha \gamma}=\sigma^{\alpha \beta}+\sigma^{\beta \gamma} .
$$

The wetting transition occurs exactly when the equality sets in (if the transition is from the partially wet to the wet state). Using the expression for the surface tension in Eq. (3.2), where the coordinate $z$ is perpendicular to each of the individual interfaces (far from the contact line region) in turn, and with the appropriate boundary conditions given by the values of the density sets $\left(\rho_{1}, \rho_{2}\right)$ in the bulk phases (Eqs. (2.3) $-(2.5))$, the conjugate gradient method is used to obtain the surface tensions of the three interfaces as functions of the field $b(\epsilon=0$ at three phase coexistence and $c=-0.7)$. The method determines values for $b$ at which the partially wet or the wet states are stable. The value of $b$ at the wetting transition, $b_{w}$, is obtained when

$$
\sigma^{\alpha \gamma}\left(b_{w}\right)=\sigma^{\alpha \beta}\left(b_{w}\right)+\sigma^{\beta \gamma}\left(b_{w}\right) .
$$

This procedure gives $b_{w}=0.6175$.

The prewetting line is expected to approach the wetting transition at $b_{w}=0.6175$ and $\epsilon=0$ tangentially, ${ }^{3}$ but such an approach is not discernible since the predicted tangency is only logarithmic., ${ }^{3,23}$ If our data points for the prewetting line are extrapolated linearly to $\epsilon=0$, the extrapolated value of $b$ at the wetting transition is $b_{w}^{e x t}=0.616$, which is shown as a filled circle on the three phase coexistence line $(\epsilon=0)$. The value of $b_{w}^{e x t}$ is smaller than the value of $b_{w}$ obtained with the conjugate gradient method. This suggests that a tangential approach of the prewetting line to the three-phase coexistence line is plausible for this model, but not discernible for the reason given above. The filled circle at the other end of the prewetting line represents the location of the prewetting critical point. Its value is obtained graphically from the mean-field behaviour of the density $\rho_{2}$ :

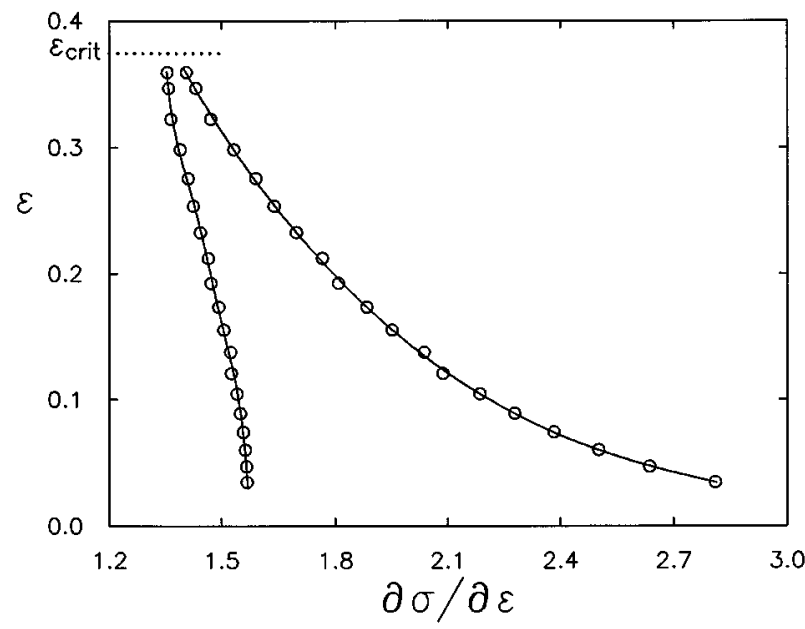

FIG. 5. Plot of the coexistence curve of two surface phases at the prewetting transition. The dotted line represents the value of $\epsilon$ at the prewetting critical point, $\boldsymbol{\epsilon}_{\text {crit }}=0.3745$.

$$
\left.\left(\rho_{2}^{\alpha \gamma *}-\rho_{2}^{\alpha \gamma}\right)\right|_{z=0} \sim\left(\epsilon_{\text {crit }}-\epsilon\right)^{\beta},
$$

and

$$
\left.\left(\rho_{2}^{\alpha \gamma *}-\rho_{2}^{\alpha \gamma}\right)\right|_{z=0} \sim\left(b-b_{\text {crit }}\right)^{\beta},
$$

at the prewetting line near the critical point. The exponent $\beta$ is the critical exponent, whose mean-field value is $1 / 2$.

Using the above two expressions to graphically determine the values of $b$ and $\epsilon$ (for $c=-0.7$ ) at the prewetting critical point, we obtain

$$
\begin{aligned}
b_{\text {crit }} & =0.3993, \\
\epsilon_{\text {crit }} & =0.3745 .
\end{aligned}
$$

The expressions in Eqs. (3.7) and (3.8) are suitable forms to accurately determine $b_{\text {crit }}$ and $\boldsymbol{\epsilon}_{\text {crit }}$. However, they are not the physical representation of the coexistence curves, since the difference $\rho_{2}^{\alpha \gamma *}-\rho_{2}^{\alpha \gamma}$ at $z=0$, which is a measure of the distance to the prewetting critical point, is chosen arbitrarily and for convenience. The "physical" coexistence curve is a plot of a thermodynamic density versus a thermodynamic field. In a two-dimensional phase equilibrium, the density is a surface density or adsorption (see Appendix 1 of Ref. 22 for a review of these terms). Using the prewetting line equation given in Eq. (3.3), and from the Gibbs adsorption equation, ${ }^{22}$ one representation of the coexistence curve would be a plot of $\epsilon$ versus $\partial \sigma / \partial \epsilon$, shown here in fig. 5. The dotted line in that figure represents the value of $\epsilon$ at the prewetting critical point. The lack of data points for the coexistence curve as $\epsilon \rightarrow 0$ and $\epsilon \rightarrow \epsilon_{\text {crit }}$ is due to the inacurracy of the calculation and is discussed in Sec. IV.

Far from the boundary region $(x \rightarrow \pm \infty)$, when two surface phases coexist, the density profile pair $\left(\rho_{1}^{\alpha \gamma}(z), \rho_{2}^{\alpha \gamma}(z)\right)$ that minimizes the surface tension $\sigma^{\alpha \gamma}$ and the profile pair $\left(\rho_{1}^{\alpha \gamma *}(z), \rho_{2}^{\alpha \gamma *}(z)\right)$ that minimizes the surface tension $\sigma^{\alpha \gamma *}$, determine the structures of the $\alpha \gamma$ and $\alpha \gamma^{*}$ interfaces, respectively. In fig. 6, we show the density profiles $\rho_{1}^{\alpha \gamma}(z)$ at $x \rightarrow-\infty$ (solid curve) and $\rho_{1}^{\alpha \gamma *}(z)$ at $x \rightarrow \infty$ (dashed curve), for $b=0.59, \quad \epsilon=0.02324$ and 


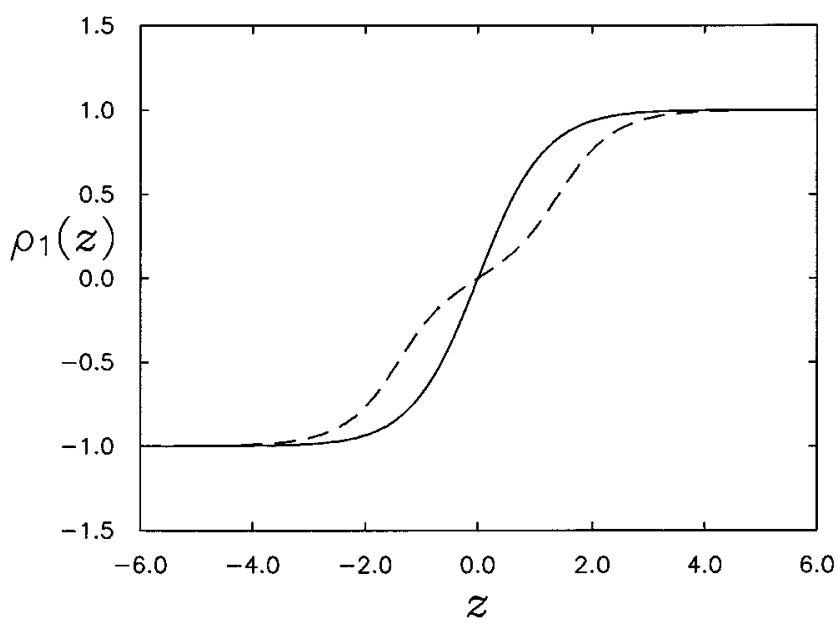

FIG. 6. Density profile $\rho_{1}(z)$ of the $\alpha \gamma *$ interface at $x \rightarrow \infty$ (dashed curve) and of the $\alpha \gamma$ interface at $x \rightarrow-\infty$ (solid curve), for $b=0.59, c=-0.7$ and $\epsilon=0.02324$.

$c=-0.7$. The density profiles $\rho_{2}^{\alpha \gamma}(z)$ at $x \rightarrow-\infty$ and $\rho_{2}^{\alpha \gamma *}(z)$ at $x \rightarrow \infty$ are shown as the solid and dashed curves respectively in fig. 7 , for the same set of fields $b, \epsilon$ and $c$ as in fig. 6.

If one eliminates the $z$ variable between $\rho_{1}(z)$ and $\rho_{2}(z)$, for a given surface phase, one obtains a trajectory in the $\rho_{1}, \rho_{2}$ plane that describes how $\rho_{1}$ and $\rho_{2}$ vary with each other through the inhomogeneous region (the surface phase) from one bulk phase to the other. In fig. 3a, we have plotted the two trajectories (dashed curves) that represent the two different structures of the $\alpha \gamma$ interface, coexisting at the prewetting transition, in the $\rho_{1}, \rho_{2}$ plane for $b=0.50$, $\epsilon=0.1553$ and $c=-0.7$. These trajectories are plotted on top of the contour lines (solid curves) of the model excess free-energy density $F$, for the same values of the thermodynamic fields. The contour lines represent the lines of constant free-energy density. The three minima in $F$ are given by the values of $\rho_{1}$ and $\rho_{2}$ in Eqs. (2.3)-(2.5). They are located in

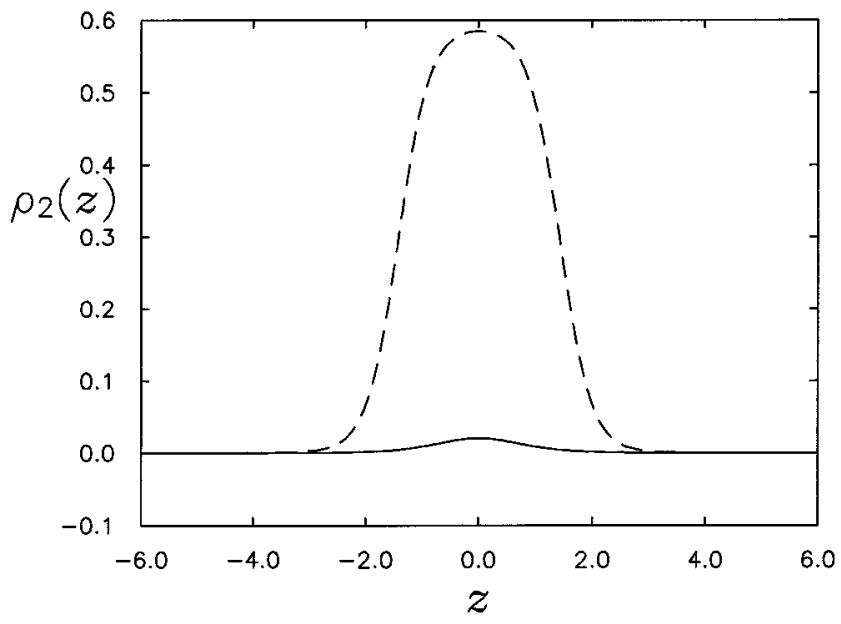

FIG. 7. Density profile $\rho_{2}(z)$ of the $\alpha \gamma *$ interface at $x \rightarrow \infty$ (dashed curve) and of the $\alpha \gamma$ interface at $x \rightarrow-\infty$ (solid curve), for $b=0.59, c=-0.7$ and $\epsilon=0.02324$. the middle of the three elliptical contour lines in fig. 3a. The upper trajectory in that figure describes the $\alpha \gamma^{*}$ surface phase, while the lower trajectory describes the $\alpha \gamma$ surface phase. In figs. $8 \mathrm{a}-8 \mathrm{c}$, we have plotted the trajectories and contours of $F$ for $b=0.46, \epsilon=0.2326$ and $c=-0.7$ in fig. $8 \mathrm{a} ; b=0.42, \epsilon=0.3227$ and $c=-0.7$ in fig. 8b; and $b=b_{\text {crit }}=0.3993, \epsilon=\epsilon_{\text {crit }}=0.3745$ and $c=-0.7$ in fig. 8c. These plots clearly demonstrate that on approach to the prewetting critical point, the two surface phases $\alpha \gamma$ and $\alpha \gamma *$ are becoming more and more alike, to become identical at the prewetting critical point (fig. 8c). This phenomenon would not have been observed had we taken the parameter $c$ to be 0 .

As a comparison, we do the same analysis as the one above for a model, studied by Blokhuis ${ }^{19}$ and Perković, Blokhuis and Han, ${ }^{16}$ that describes a fluid on a substrate whose interface might become wet by another fluid phase. We study the density profiles of the two surface phases in coexistence at the prewetting transition. The substrate is treated as a boundary condition which makes the system effectively of one component, of density $\rho$. There are three thermodynamic fields $h_{1}, g$ and $h$ which are analogous to $b$, $c$ and $\epsilon$, respectively. The field $h_{1} \geqslant 0, g=0$ (for convenience) and $h>0$, measures the distance from the threephase coexistence line.

In fig. 9, we show a plot of the difference between the density profiles of the two surface phases far from the boundary line region, as a function of the coordinate $z$ : $\Delta \rho(z) \equiv \rho^{\alpha \gamma *}(z)-\rho^{\alpha \gamma}(z)$. As the prewetting critical point is approached, $\quad h \rightarrow h_{\text {crit }}=\frac{4}{9}(3)^{1 / 2} \sim 0.7698, \quad(g=0 \quad$ and $h_{1, \text { crit }}=3^{1 / 2}$ ), the two surface phases become more alike, and their structures become identical at the prewetting critical point (as seen by the approach of $\Delta \rho(z)$ to the value of 0 ).

\section{BOUNDARY TENSION}

At the prewetting transition, the two different structures of the $\alpha \gamma$ interface coexist by creating a one-dimensional boundary between themselves (fig. 1). Associated with that boundary line is the tension $\tau_{b}$, cf. Eq. (1.1),

$$
\tau_{b}=\min _{\rho_{1}, \rho_{2}}\left[\int_{-\infty}^{\infty} d x\left[\int_{-\infty}^{\infty} d z \quad \Psi\left(\rho_{1}, \rho_{2}\right)\right]-\sigma\right],
$$

where the density profiles $\rho_{1}$ and $\rho_{2}$ are now twodimensional functions $\rho_{1}(x, z)$ and $\rho_{2}(x, z)$; the coordinate $x$ is parallel to the $\alpha \gamma$ interface and the coordinate $z$ is perpendicular to it (fig. 1); $\Psi\left(\rho_{1}, \rho_{2}\right)$ is given as in Eq. (2.1) and $\sigma$ is the surface tension of the coexisting interfaces at the prewetting transition as given by Eq. (3.2). As in the previous section, the distances, densities and free energies are scaled so that they are all dimensionless.

Minimization of Eq. (4.1) with respect to $\rho_{1}$ and $\rho_{2}$ yields two Euler-Lagrange equations,

$$
\begin{aligned}
& \nabla^{2} \rho_{1}=\frac{\partial F}{\partial \rho_{1}}, \\
& \nabla^{2} \rho_{2}=\frac{\partial F}{\partial \rho_{2}},
\end{aligned}
$$




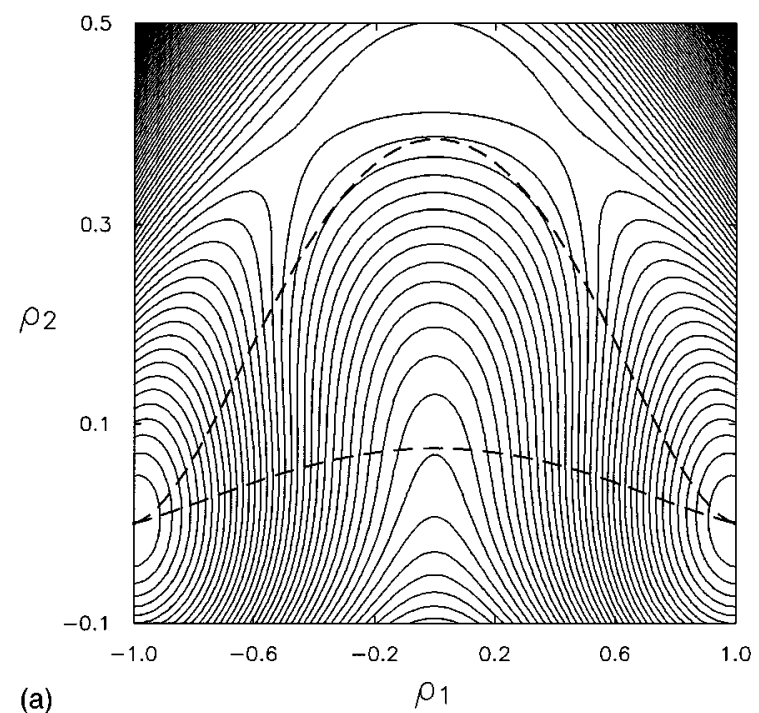

(a)

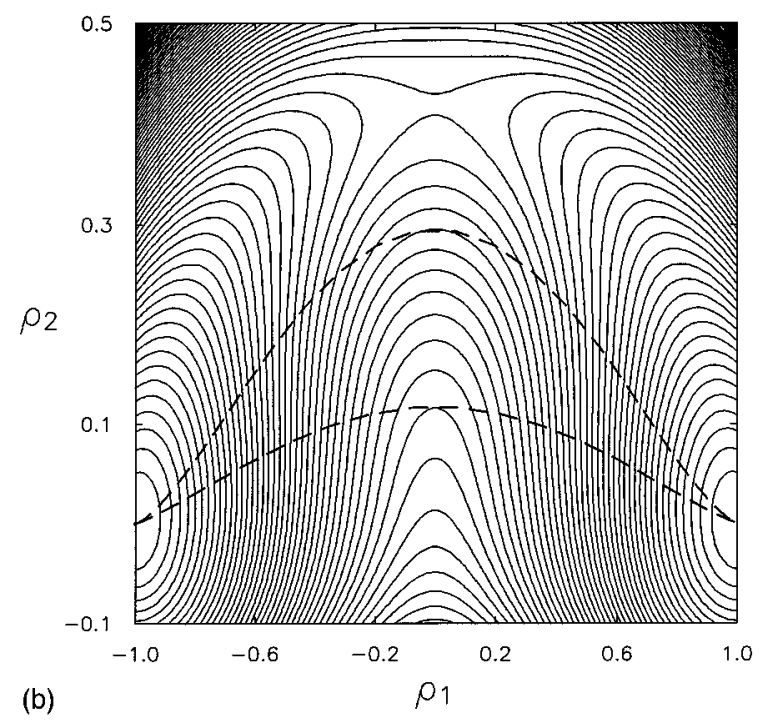

(b)

where $F$ is given in Eq. (2.2) and the $\nabla$ gradient operator is two-dimensional in the $x$ and $z$ directions. The boundary conditions are given by the values of the densities $\rho_{1}$ and $\rho_{2}$ at $z \rightarrow \pm \infty$ :

$$
\left(\rho_{1}, \rho_{2}\right)= \begin{cases}(1,0), & z \rightarrow+\infty \\ (-1,0), & z \rightarrow-\infty\end{cases}
$$

and the density profile pairs $\left(\rho_{1}(z), \rho_{2}(z)\right)$ at $x \rightarrow \pm \infty$. At $x \rightarrow+\infty$, the density profiles $\rho_{1}(z)$ and $\rho_{2}(z)$ are the profiles that describe the $\alpha \gamma *$ interface at the prewetting transition (dashed curves in fig. 6 and fig. 7 respectively). Similarly, at $x \rightarrow-\infty$, the boundary condition is given by the density profiles $\rho_{1}(z)$ and $\rho_{2}(z)$ of the $\alpha \gamma$ interface at the prewetting transition (solid curves in fig. 6 and fig. 7 respectively).

The integral in Eq. (4.1) converges sufficiently fast as $x, z \rightarrow \pm \infty$ so that we can replace the infinite limits of integration with large, finite limits. Then, the area of integration becomes a large, finite domain (fig. 1), where the system of the two Euler-Lagrange equations ((4.2) and (4.3)) needs to be solved for the densities $\rho_{1}(x, z)$ and $\rho_{2}(x, z)$.

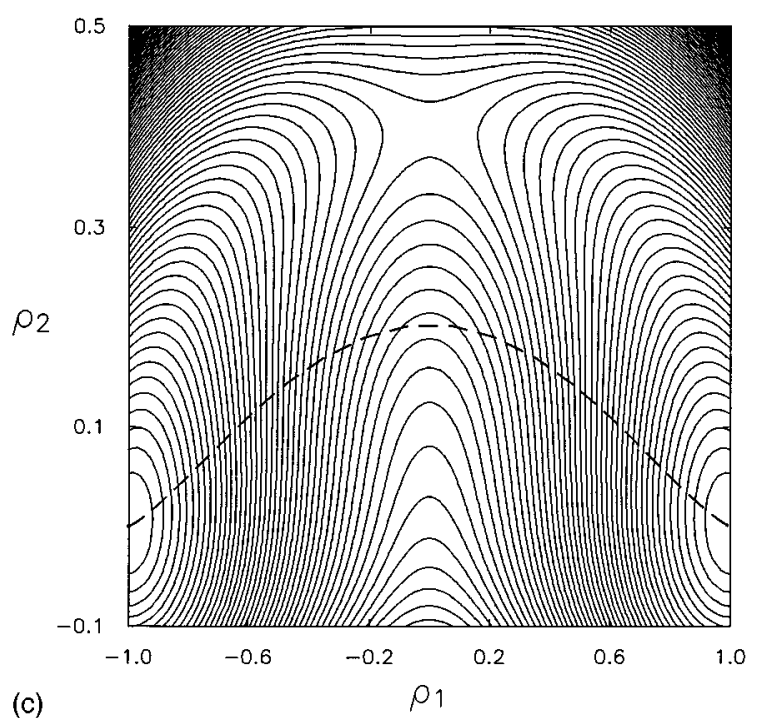

FIG. 8. Plots of the contours of $F$ from Eq. (2.2) (solid curves) with two trajectories (dashed curves) representing coexistence of the two fluid surface phases at the prewetting transition for $c=-0.7$ and (a) $b=0.46$, $\epsilon=0.2326 ; \quad$ (b) $\quad b=0.42, \quad \epsilon=0.3227 ; \quad$ (c) $\quad b=b_{\text {crit }}=0.3993$, $\epsilon=\epsilon_{c r i t}=0.3745$. The two trajectories become identical at the prewetting critical point.

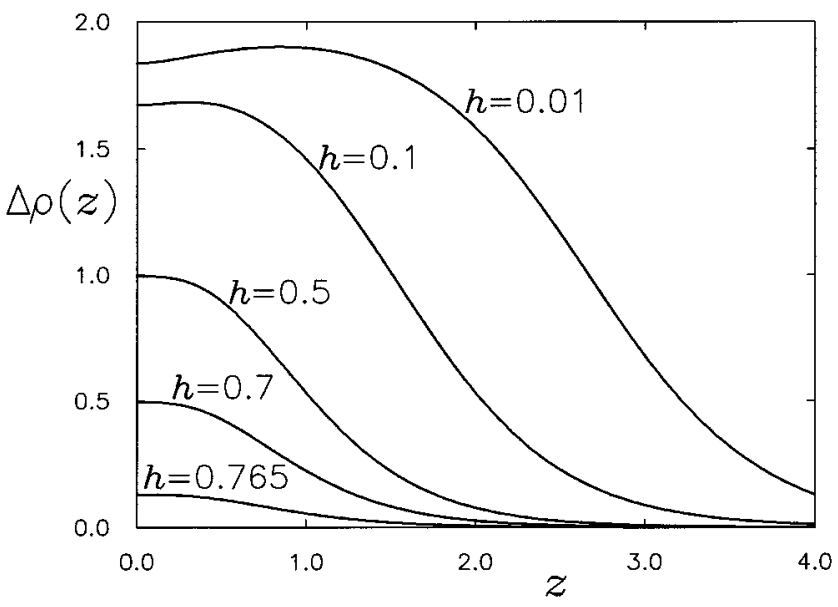

FIG. 9. A plot of the difference between the two surface phase density profiles for a system of one fluid phase on a substrate, as a function of $z$, for various values of the thermodynamic field $h$. The prewetting critical point occurs at $h_{\text {crit }}=\frac{4}{9}(3)^{1 / 2} \sim 0.7698$ and $h_{1, \text { crit }}=3^{1 / 2}$, when $g=0$ (Ref. 19). 


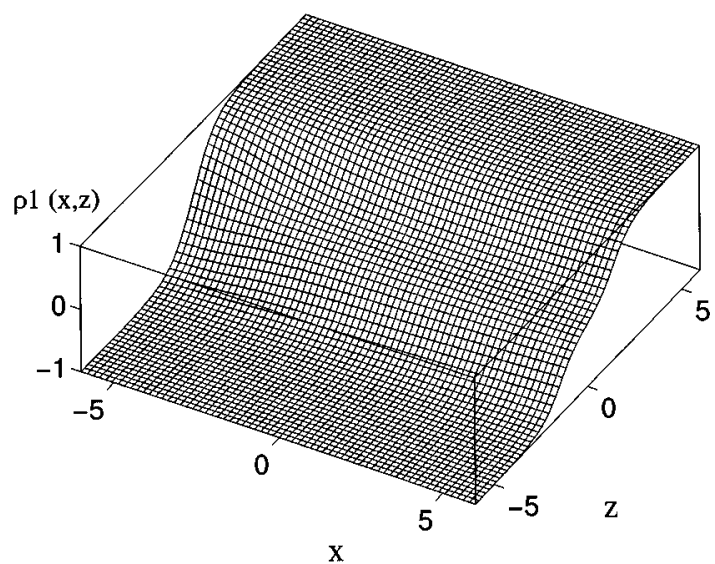

FIG. 10. The density profile $\rho_{1}(x, z)$ at the prewetting transition, for $b=0.59, c=-0.7$ and $\epsilon=0.02324$.

Equations (4.2) and (4.3) represent a system of two nonlinear partial differential equations with four Dirichlet boundary conditions (eq. (4.4) and the numerical profiles $\rho_{1}(z)$ and $\rho_{2}(z)$ at $\left.x \rightarrow \pm \infty\right)$. We have used a multigrid algorithm to solve the above system for the densities $\rho_{1}(x, z)$ and $\rho_{2}(x, z)$. The multigrid method is based on discretizing the partial differential equations on grids of different levels of coarseness. The system is iterated on the finest grid using a traditional iteration (smoothing) method such as a GaussSeidel method, until the iterations become very slowly convergent. To speed-up the convergence rate, the density profiles are transferred onto the next coarser grid via a restriction operator, and the smoothing procedure is continued. The restriction to coarser grids is continued until the iterations converge or the coarsest grid is reached where the solution to the discretized system of equations can be easily obtained. Then, the density profiles are brought back to the finest grid via a prolongation operator. This method has been successfully applied in the calculation of the boundary and line tensions in a system of two fluid phases on a substrate. ${ }^{16}$ There, however, only one non-linear partial differential equation had to be solved for the density $\rho$ of the system, with one Neumann and three Dirichlet boundary conditions.

As in Ref. 16 we use the Full Approximation Storage Algorithm (FAS). ${ }^{21}$ The smoothing at each grid level is achieved with a red-black Gauss-Seidel relaxation method. The restriction operator uses a half-weighting restriction, while the prolongation operator is a bilinear interpolation. The domain over which the two density profiles $\rho_{1}(x, z)$ and $\rho_{2}(x, z)$ are determined is a square with $257 \times 257$ grid points. Since the prolongation operator is a bilinear interpolation, the number of points in one dimension is $2^{N G}+1$, where $N G$ is the number of discretization grids; in our case $N G=8$. Due to that constraint, the domain size and the grid spacing $h_{s}$ are not independent of each other. The largest grid spacing used is $h_{s}=0.075$.

In fig. 10 and fig. 11, we show examples of the twodimensional density profiles, $\rho_{1}(x, z)$ and $\rho_{2}(x, z)$ respectively, for $b=0.59, c=-0.7$ and $\epsilon=0.02324$, obtained as the solutions of the two Euler-Lagrange equations in Eqs. (4.2) and (4.3). Using such density profiles, and with the

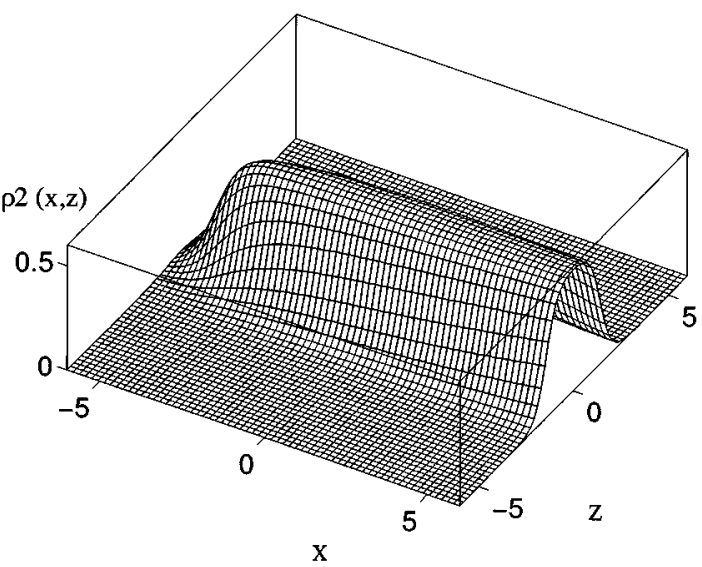

FIG. 11. The density profile $\rho_{2}(x, z)$ at the prewetting transition, for $b=0.59, c=-0.7$ and $\epsilon=0.02324$.

corresponding values for the fields $b, c$ and $\epsilon$, the boundary tension $\tau_{b}$ is calculated from Eq. (4.1). The open circles in fig. 12 represent the data for the boundary tension $\tau_{b}$ versus $\epsilon$, the thermodynamic field that is a measure of the distance from the three-phase coexistence curve. The value of $c$ is fixed at $c=-0.7$ and the value of $b$ is obtained from the prewetting line (fig. 4). The solid curve is a smooth interpolation between the data points. The error bars will be discussed below.

As the wetting transition is approached $(\epsilon \rightarrow 0)$, the boundary tension increases in magnitude. The increase is due to an increase of the inhomogeneous region where the two surface phases meet, as the $\alpha \gamma^{*}$ surface phase becomes thicker. In order to obtain a value for $\tau_{b}$ at the wetting transition, we fit the four data points for the boundary tension closest to the wetting transition with the following form:

$$
\tau_{b}=\tau_{b, w}-\tau_{+} \epsilon^{1 / 2},
$$

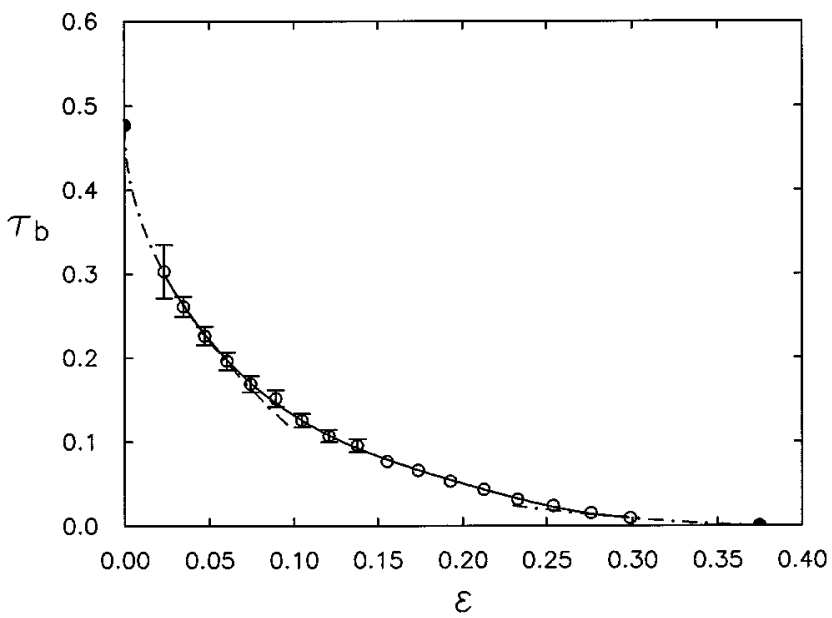

FIG. 12. Plot of the boundary tension $\tau_{b}$ versus $\epsilon$. The prewetting critical point is at $\epsilon_{c r i t}=0.3745$ (filled circle), where $\tau_{b}=0$. The value of $\tau_{b}$ at the wetting transition $(\epsilon=0)$ is $\tau_{b}=0.477$ (filled circle). The error bars are the standard deviation from the mean, calculated as described in the text. The dash-dot-dash fits are described in the text as well. 
where $\tau_{b, w}$ and $\tau_{+}$are fitting parameters. This form is analogous to the expression for $\tau_{b}$ close to the wetting transition, determined by Indekeu $^{17}$ in the interface displacement model, and by Varea and Robledo in numerical studies, ${ }^{18}$ for a system of one fluid phase on a substrate (see also Ref. 16).

The expression in Eq. (4.5) is valid only for very small values of $\epsilon$. The data points over which the fit is done are assumed to meet that requirement, even though that is probably not accurate. The fitting parameters are obtained as:

$$
\begin{aligned}
& \tau_{b, w}=0.477, \\
& \tau_{+}=1.15 .
\end{aligned}
$$

Therefore, at the wetting transition, the boundary tension $\tau_{b}$ is finite and is equal to $\tau_{b, w}=0.477$. The expression in Eq. (4.5), with the values for $\tau_{b, w}$ and $\tau_{+}$given by Eqs. (4.6) and (4.7) respectively, is plotted as the dash-dot-dash curve close to the wetting transition $(\epsilon \rightarrow 0)$, in fig. 12. The filled circle is at $\tau_{b, w}$. If we choose to fit five data points, rather than four, with the same form as in Eq. (4.5), the value of the boundary tension at the wetting transition is $\tau_{b, w}=0.470$.

Moving along the prewetting line from the wetting transition $(\epsilon=0)$ towards the prewetting critical point (filled circle at $\epsilon=0.3745$ ), where the two surface phases become indistinguishable (see Sec. III), the boundary tension $\tau_{b}$ decreases in magnitude from $\tau_{b, w}=0.477$ to $\tau_{b}=0$. The boundary tension vanishes at the prewetting critical point for the same reason that the surface tension of an interface vanishes at bulk criticality, since the boundary line is the twodimensional analogue of an interface in a three-dimensional system. For that same reason, the boundary tension $\tau_{b}$ is always positive. Due to this analogy, the boundary tension $\tau_{b}$ close to the prewetting critical point scales as

$$
\tau_{b}=A\left|\left(\epsilon_{\text {crit }}-\epsilon\right) / \epsilon_{\text {crit }}\right|^{\mu},
$$

where $\left(\epsilon_{\text {crit }}-\epsilon\right) / \epsilon_{\text {crit }}$ is a reduced field that measures the distance from the prewetting critical point, $A$ is a proportionality constant and $\mu$ is the critical point exponent. Nakanishi and Fisher ${ }^{24}$ conjectured from a theoretical analysis that the prewetting critical point exhibits 2D Ising-like criticality. This conjecture was verified by Nicolaides and Evans ${ }^{5}$ in Monte Carlo simulations. Experimental study of the prewetting critical region of the binary liquid mixture methanolcyclohexane by Kellay, Bonn and Meunier ${ }^{12}$ further verified the 2D Ising-like character of the prewetting critical point. Therefore, $\mu=1$. This exponent was verified experimentally by Benvegnu and McConnell, ${ }^{20}$ when they measured the tension between lipid monolayers at the air-water interface. Our model, however, is mean-field and we expect that the boundary tension $\tau_{b}$ close to the prewetting critical point has a mean-field critical point exponent $\mu=\frac{3}{2}$. We conjecture such a behaviour and determine the proportionality constant $A$ from the value of a single data point, the one closest to the prewetting critical point $\epsilon_{\text {crit }}=0.3745$. We obtain $A=0.098$. As in the fit close to the wetting transition, the assumption is that the data point used is close enough to the prewetting critical point to be in the asymptotic regime, even though that is probably not accurate. The expression in Eq. (4.8) is plotted in fig. 12 as the dash-dot-dash curve near the prewet- ting critical point. The reason for not having more data points close to the two limiting regimes of wetting and criticality will be discussed later.

We now determine orders of magnitude for our dimensionless boundary tensions by scaling them with a typical force $k T / d$, where $k$ is Boltzmann's constant, $T$ is the temperature and $d$ is a typical length. We choose $T=300 \mathrm{~K}$, and a unit of length comparable to the order of molecular dimensions, $d=10 \AA$. We obtain values for the boundary tension going from 0 at the prewetting critical point to values of $Q\left(10^{-12}\right) \mathrm{N}$, close to the wetting transition. This is the range of values that Benvegnu and McConnell ${ }^{20}$ obtained from their experimental determination of the boundary tension of the boundary between two lipid monolayer domains at the air-water interface. In their work, they measured the boundary tension as a function of a surface pressure $\Pi$, defined as the difference between the surface tension of the air-water interface and the surface tension of the air-water interface containing a lipid monolayer. When the surface pressure $\Pi=0$, the air-water interface has no lipid monolayer on it. Such a state is analogous to bulk phase coexistence in our model, when $\epsilon=0$, when there is no surface phase coexistence. Benvegnu and $\mathrm{McConnell}^{20}$ also determined the value of $\Pi$ at the prewetting critical point, $\Pi_{\text {crit }}=10.5 \mathrm{dyne} / \mathrm{cm}$, which corresponds to the same state as described by $\epsilon_{\text {crit }}=0.3745$. Therefore, the reduced phenomenological parameter $\left(\epsilon_{\text {crit }}-\epsilon\right) / \epsilon_{\text {crit }}$ can be viewed as a reduced surface pressure $\left(\Pi_{\text {crit }}-\Pi\right) / \Pi_{\text {crit }}$, since the two limiting cases of wetting $(\epsilon \rightarrow 0)$ and criticality can be mapped onto the two limiting physical cases of an interface with no monolayer on it $(\Pi \rightarrow 0)$ and 2D criticality, respectively. However, this type of comparison is only qualitatively correct since the experimental system studied by Benvegnu and $\mathrm{McConnell}^{20}$ includes the presence of long-ranged dipole interactions and the boundary tension shows 2D Ising-like behaviour, close to the prewetting critical point. Our model, on the other hand, is mean-field and is restricted to short-ranged forces.

Using the same units of force and length as in the above case, we obtain orders of magnitude for the boundary tension $\tau_{b}$ in the system of a fluid phase on a substrate as well. ${ }^{16}$ The boundary tension is of $\mathscr{O}\left(10^{-12}\right) \mathrm{N}$ close to the wetting transition, and goes to 0 at the prewetting critical point.

In order to determine the accuracy of the boundary tension calculation using the multigrid method, we have calculated the boundary tension $\tau_{b}$ using two other formulas (Appendix A), in addition to Eq. (4.1). One of them is the Kerins-Boiteux formula for the line tension: ${ }^{25}$

$\tau_{b}^{K-B}=\int_{-\infty}^{\infty} d x \int_{-\infty}^{\infty} d z\left[\frac{1}{2}\left(\nabla \rho_{1}\right)^{2}+\frac{1}{2}\left(\nabla \rho_{2}\right)^{2}-F\left(\rho_{1}, \rho_{2}\right)\right]$,

and the other formula is:

$$
\tau_{b}=\int_{-\infty}^{\infty} d x \int_{-\infty}^{\infty} d z\left[\left(\frac{\partial \rho_{1}}{\partial x}\right)^{2}+\left(\frac{\partial \rho_{2}}{\partial x}\right)^{2}\right] .
$$

The integrals in Eqs. (4.9) and (4.10) are not variational integrals, i.e., the values of the boundary tensions obtained from these two integrals are not extrema when $\rho_{1}(x, z)$ and $\rho_{2}(x, z)$ are the equilibrium profiles. 
The boundary tensions are calculated by substituting $\rho_{1}(x, z)$ and $\rho_{2}(x, z)$, obtained from the multigrid method, in Eq. (4.9) and (4.10). The difference in the values of $\tau_{b}$ obtained from Eqs. (4.1), (4.9) and (4.10) describes qualitatively how close the density profiles $\rho_{1}(x, z)$ and $\rho_{2}(x, z)$ obtained from the multigrid algorithm are to the equilibrium density profiles: if the boundary tensions obtained from Eqs. (4.1), (4.9) and (4.10) are equal, the multigrid density profiles are the equilibrium profiles. The error bars in fig. 12 represent the standard deviation from the average of the values of $\tau_{b}$ from Eqs. (4.1), (4.9) and (4.10). These error bars are centered at the values of $\tau_{b}$ obtained from Eq. (4.1), since that equation is the one associated with the Euler-Lagrange equations (4.2) and (4.3) whose solutions give the exact (within the numerical accuracy) density profiles $\rho_{1}(x, z)$ and $\rho_{2}(x, z)$, and hence give the best estimate for the boundary tension $\tau_{b}$. If no error bars are shown in fig. 12, the standard deviation is within the size of the data point.

As the wetting transition is approached $(\epsilon \rightarrow 0)$, the accuracy in the calculation of the boundary tension decreases, as suggested by the large error bars (fig. 12). This is due to the increase in the inhomogeneous boundary area as the $\alpha \gamma *$ interface becomes thicker. Therefore, the domain over which the Euler-Lagrange equations (4.2) and (4.3) have to be solved is increased, leading to a larger grid spacing $h_{s}$, since the number of grid points cannot be increased proportionally due to computation time constraints. Consequently, the accuracy in the calculated values of the boundary tension decreases. Close to the prewetting critical point, the accuracy decreases as well, but is not reflected by large error bars. This is due to the very small values for the boundary tension. Therefore, errors in $\tau_{b}$ of the same order of magnitude as $\tau_{b}$ will not be larger than the size of the data points in fig. 12 . In this case, however, the inaccuracy in the boundary tension values occurs due to the increase in the domain size along the $x$-axis only. This is in contrast to the domain increase in both the $x$ - and $z$-directions close to the wetting transition.

\section{CONCLUSION}

In this paper, we have presented numerically exact calculations of the boundary tension $\tau_{b}$ of the one-dimensional boundary that is created when two surface phases meet edge-on at the prewetting transition, in a system of two bulk fluid phases, where a third phase might become bulk. The prewetting line was determined from the wetting transition to the prewetting critical point. Close to the wetting transition, the boundary tension increases in magnitude and extrapolates to a finite value at the wetting transition, $\tau_{b, w}=0.477$, if one assumes the asymptotic form of Indekeu. ${ }^{17}$ Close to the prewetting critical point, the boundary tension vanishes in a conjectured mean-field manner, with the critical point exponent $\mu=3 / 2$. Scaling of the boundary tensions with a typical unit of force yields an order of magnitude for $\tau_{b}$ of $10^{-12} \mathrm{~N}$, close to the wetting transition, and decreasing tensions to 0 at the prewetting critical point. As the prewetting critical point is approached, the two surface phases become more and more alike, and become indentical at the prewetting critical point.

\section{ACKNOWLEDGMENTS}

We would like to thank I. Szleifer for his generous offer to use his computing equipment as well as for useful discussions, and D.J. Bukman for useful discussions and a careful reading of this manuscript. The research of E.M.B. has been made possible by a fellowship of the Royal Netherlands Academy of Arts and Sciences. This work was supported by the National Science Foundation and the Cornell University Materials Science Center.

\section{APPENDIX. KERINS-BOITEUX FORMULA FOR THE BOUNDARY TENSION}

In this appendix, we derive two different, but equivalent, formulas for the boundary tension $\tau_{b}$ along the prewetting line, for the general case of an $n$-component two-phase system. For a 2-component system, one of the formulas is the Kerins-Boiteux formula ${ }^{25}$ for the line tension of three fluid phases. It turns out that the same expression is valid for the boundary tension as well.

The boundary tension is given by, cf. Eq. (4.1),

$$
\begin{aligned}
\tau_{b}= & \int_{-\infty}^{\infty} d x\left[\int_{-\infty}^{\infty} d z\left[\sum_{i=1}^{n} \frac{1}{2}\left(\nabla \rho_{i}\right)^{2}+F\left(\rho_{1}, \rho_{2}, \ldots, \rho_{n}\right)\right]\right. \\
& -\sigma],
\end{aligned}
$$

where the density profiles $\rho_{i}=\rho_{i}(x, z)$ are solutions of the Euler-Lagrange equations, cf. Eq. (4.2),

$$
\nabla^{2} \rho_{i}=\frac{\partial F}{\partial \rho_{i}}, \quad i=1,2, \ldots, n
$$

with the boundary conditions given by the values of the densities $\rho_{i}$ in the bulk phases and the density profiles $\rho_{i}(z)$ across the interface, at $x \rightarrow \pm \infty$.

Multiplying both sides of the Euler-Lagrange equation in (A2) by $\partial \rho_{i} /(\partial x)$, adding all the equations for $i=1,2, \ldots, n$ together and integrating over $z$ from $-\infty$ to $\infty$ gives

$$
\int_{-\infty}^{\infty} d z\left[\sum_{i=1}^{n}\left[\frac{\partial^{2} \rho_{i}}{\partial x^{2}} \frac{\partial \rho_{i}}{\partial x}+\frac{\partial^{2} \rho_{i}}{\partial z^{2}} \frac{\partial \rho_{i}}{\partial x}-\frac{\partial F}{\partial \rho_{i}} \frac{\partial \rho_{i}}{\partial x}\right]\right]=0
$$

Next, we partially integrate the second term and write the other terms as derivatives,

$$
\begin{aligned}
\int_{-\infty}^{\infty} d z & {\left[\sum_{i=1}^{n}\left[\frac{1}{2} \frac{\partial}{\partial x}\left(\frac{\partial \rho_{i}}{\partial x}\right)^{2}-\frac{\partial \rho_{i}}{\partial z} \frac{\partial^{2} \rho_{i}}{\partial x \partial z}\right]\right.} \\
\left.-\frac{\partial}{\partial x} F\left(\rho_{1}, \rho_{2}, \ldots, \rho_{n}\right)\right] & =-\left[\sum_{i=1}^{n} \frac{\partial \rho_{i}}{\partial z} \frac{\partial \rho_{i}}{\partial x}\right]_{z=-\infty}^{z=\infty}=0,
\end{aligned}
$$

where we have used the boundary conditions that the densities $\rho_{i}$ are constant in the bulk phases (at $z \rightarrow \pm \infty$ ), to derive the last identity. 
We now write the second term in the sum on the left hand side of Eq. (A4) as a derivative, integrate over $x$ from $x^{\prime}$ to $\infty$ and subsequently drop the prime. We are then finally left with

$$
\begin{aligned}
\int_{-\infty}^{\infty} d z & {\left[\sum_{i=1}^{n}\left[\frac{1}{2}\left(\frac{\partial \rho_{i}}{\partial x}\right)^{2}-\frac{1}{2}\left(\frac{\partial \rho_{i}}{\partial z}\right)^{2}\right]-F\left(\rho_{1}, \rho_{2}, \ldots, \rho_{n}\right)\right] } \\
=-\sigma &
\end{aligned}
$$

where $\sigma=\int_{-\infty}^{\infty} d z \quad\left[F\left(\rho_{1}, \rho_{2}, \ldots, \rho_{n}\right)+\sum_{i=1}^{n} \frac{1}{2}\left(d \rho_{i} / d z\right)^{2}\right]$.

A similar formula can be derived by performing an analysis analogous to the one above, but now one multiplies both sides of the Euler-Lagrange equation in Eq. (A2) by $\partial \rho_{i} /(\partial z)$ and integrates over $x$ from $-\infty$ to $\infty$. The analysis follows exactly the same steps as the analysis above, so we only give the final result:

$$
\begin{gathered}
\int_{-\infty}^{\infty} d x\left[\sum_{i=1}^{n}\left[-\frac{1}{2}\left(\frac{\partial \rho_{i}}{\partial x}\right)^{2}+\frac{1}{2}\left(\frac{\partial \rho_{i}}{\partial z}\right)^{2}\right]\right. \\
\left.-F\left(\rho_{1}, \rho_{2}, \ldots, \rho_{n}\right)\right]=0 .
\end{gathered}
$$

Integrating Eq. (A5) over $x$ from $-\infty$ to $\infty$ and Eq. (A6) over $z$ from $-\infty$ to $\infty$ and adding the results to the expression for the boundary tension in Eq. (A1) leaves us with the KerinsBoiteux formula for $n$ components:

$$
\tau_{b}^{K-B}=\int_{-\infty}^{\infty} d x \int_{-\infty}^{\infty} d z\left[\sum_{i=1}^{n} \frac{1}{2}\left(\nabla \rho_{i}\right)^{2}-F\left(\rho_{1}, \rho_{2}, \ldots, \rho_{n}\right)\right] .
$$

For $n=2$, we recover the formula in Eq. (4.9).

If one integrates Eq. (A5) over $x$ from $-\infty$ to $\infty$ and adds the result to the expression for the boundary tension in Eq. (A1), one obtains

$$
\tau_{b}=\int_{-\infty}^{\infty} d x \int_{-\infty}^{\infty} d z\left[\sum_{i=1}^{n}\left(\frac{\partial \rho_{i}}{\partial x}\right)^{2}\right],
$$

which is the formula in Eq. (4.10), for $n=2$.

Although the formula in Eq. (A8) is even simpler than the Kerins-Boiteux formula in Eq. (A7), it is only valid as an expression for the boundary tension, whereas the formula in Eq. (A7) is also valid for the line tension along partial wetting. ${ }^{25}$

${ }^{1}$ J.W. Cahn, J. Chem. Phys. 66, 3667 (1977).

${ }^{2}$ C. Ebner and W.F. Saam, Phys. Rev. Lett. 38, 1486 (1977).

${ }^{3}$ E.H. Hauge and M. Schick, Phys. Rev. B 27, 4288 (1983).

${ }^{4}$ D. Nicolaides and R. Evans, Phys. Rev. B 39, 9336 (1989).

${ }^{5}$ D. Nicolaides and R. Evans, Phys. Rev. Lett 63, 778 (1989).

${ }^{6}$ J.E. Finn and P.A. Monson, Phys. Rev. A 39, 6402 (1989).

${ }^{7}$ E. Velasco and P. Tarazona, Phys. Rev. A 42, 2454 (1990).

${ }^{8}$ J.E. Rutledge and P. Taborek, Phys. Rev. Lett. 69, 937 (1992).

${ }^{9}$ K.S. Ketola, S. Wang, and R.B. Hallock, Phys. Rev. Lett. 68, 201 (1992).

${ }^{10}$ L. Ghaicha, M. Privat, L. Tenebre, R. Bennes, E. Tronel-Peyroz, and J.M. Douillard, Langmuir 4, 1326 (1988).

${ }^{11}$ R. Bennes, M. Privat, E. Tronel-Peyroz, and M. Amara, Langmuir 7, 1088 (1991).

${ }^{12}$ H. Kellay, D. Bonn, and J. Meunier, Phys. Rev. Lett. 71, 2607 (1993).

${ }^{13}$ J.D. van der Waals, Verhandel. Konink. Akad. Weten., Amsterdam, 1, 56 (1893); English translation in J. Stat. Phys. 20, 197 (1979).

${ }^{14}$ B. Widom and A.S. Clarke, Physica A 168, 149 (1990).

${ }^{15}$ B. Widom, in Condensed Matter Theories, edited by L. Blum and F.B. Malik (Plenum, New York, 1993), pp. 589-593.

${ }^{16}$ S. Perković, E.M. Blokhuis, and G. Han, J. Chem. Phys. 102, 400 (1995).

${ }^{17}$ J.O. Indekeu, Physica A 183, 439 (1992).

${ }^{18}$ C. Varea and A. Robledo, Phys. Rev. E 47, 3772 (1993).

${ }^{19}$ E.M. Blokhuis, Physica A 202, 402 (1994).

${ }^{20}$ D.J. Benvegnu and H.M. McConnell, J. Phys. Chem. 96, 6820 (1992).

${ }^{21}$ W.H. Press, S.A. Teukolsky, W.T. Vetterling, and B.P. Flannery, Numerical Recipes in Fortran, 2nd ed. (Cambridge University Press, Cambridge, 1992).

${ }^{22}$ J.S. Rowlinson and B. Widom, Molecular Theory of Capillarity (Clarendon, Oxford, 1982).

${ }^{23}$ S. Perković, I. Szleifer, and B. Widom, Mol. Phys. 80, 729 (1993).

${ }^{24}$ H. Nakanishi and M.E. Fisher, Phys. Rev. Lett. 49, 1565 (1982).

${ }^{25}$ J. Kerins and M. Boiteux, Physica A 117, 575 (1983). 